\title{
Preparation and Bioactivity Properties of a Novel Composite Membrane of Fructose Mediated $\beta$-Tricalcium Pyrophosphate/(Polyethylene Glycol/Chitosan) for Guided Tissue Regeneration
}

\author{
Jian-Wen Wang, ${ }^{1}$ Min-Hsiung Hon, ${ }^{2}$ Yi-Ming Kuo, ${ }^{1}$ and Mei-Hui Chung ${ }^{1}$ \\ ${ }^{1}$ Department of Environmental and Safety Engineering, Chung Hwa University of Medical Technology, Tainan 717, Taiwan \\ ${ }^{2}$ Department of Materials Science and Engineering, National Cheng Kung University, Tainan 70101, Taiwan
}

Correspondence should be addressed to Jian-Wen Wang; jinwen.tw@yahoo.com.tw

Received 26 September 2014; Accepted 15 December 2014

Academic Editor: Long Yu

Copyright (C) 2015 Jian-Wen Wang et al. This is an open access article distributed under the Creative Commons Attribution License, which permits unrestricted use, distribution, and reproduction in any medium, provided the original work is properly cited.

A novel composite membrane of $\beta$-tricalcium pyrophosphate ( $\beta$-TCP) and fructose- (F-) mediated chitosan/poly(ethylene glycol) (CS/PEG) was prepared by thermally induced phase separation technique. The prepared composite membranes were characterized using scanning electron microscopy (SEM) and X-ray diffraction (XRD). The mechanical property, swelling, degradation, and cytotoxicity of the composite membranes were evaluated in vitro with respect to its potential for use as biodegradable guided tissue regeneration (GTR) membrane. In vitro degradation tests showed the composite membrane with a controllable degradation rate when changing the $\beta$-TCP content. The incorporation of $\beta$-TCP granules also caused a significant enhancement of tensile strength. When $\beta$-TCP content is controlled to $50 \mathrm{wt} \%$, homogeneous composite membranes with well mechanical property and enzymatic degradation rate can be obtained. Cytotoxicity assay demonstrates that the composite membranes were nontoxic and had very good cell compatibility. Most importantly, the release of calcium ions and glucosamine from the composite membranes was proved to increase the cell proliferation of NIH3T3. The results of this study have indicated that this novel F- $\beta$-TCP/CS/PEG composite can be a suitable material for GTR applications.

\section{Introduction}

Guided tissue regeneration (GTR) is a technique utilizing membranes to serve as a physical barrier to separate and create a secluded space around the defects [1]. This permits bone regeneration and prevents the epithelial cells from migrating into the bony area [2]. Consequently, GTR method required the actual barrier membranes to possess good biocompatibility, biofunction, and excellent mechanical strength. Nondegradable membranes such as polytetrafluoroethylene have superior space-maintaining properties and capacity for cell occlusion which are widely used as barrier membranes. However, a second surgical procedure is required for removal. Finally, membrane exposure is frequent, increasing the risk of secondary infection [3]. Comparatively, bioresorbable membranes are more advantageous for patients as they avoid the need for secondary surgery, such as collagen, chitosan, poly(L-lactide) (PLLA), and poly(Llactide-co-glycolide) (PLGA) and copolymers are already being used in clinical practice due to their excellent cell affinity, biodegradability, and biocompatibility. Chitosan (CS), a cationic polysaccharide, is obtained by alkaline deacetylation of chitin, the principal exoskeletal component in crustaceans. In recent years, CS has been widely used in biomedical applications due to its biodegradability, nontoxicity, antibacterial activity, and biocompatibility [4]. Although CS and its derivatives seem to have very excellent properties as biomaterial, but the low degradation rate in aqueous media due to highly crystallized and rigid structure is limited to its application. Various studies were conducted to accelerate the degradation rate of CS by chemical modification techniques. However, chemical modifications will change the fundamental chemistry structure of $\mathrm{CS}$ which resulted in the reductions or loss of the original property. The blending membranes 
deserve attention because its process is simple and mild [5]. Blending water-soluble polymers such as poly(ethylene glycols) (PEGs) have proved either improvement the degradation rate or water affinity of CS membrane in our group [6]. He et al. [7] also found that the ductility of the blend films is tremendously improved due to the reduced crystallinity of CS. The disadvantages of CS are that it is mechanically brittle and lacks sufficient bioactivity to induce initial rapid bone regeneration [8]. The brittleness of the membrane is unable to provide sufficient space adjacent to the defect to allow for the regeneration of the desirable tissue into the space by precluding epithelial cells and gingival connective tissue cells which are believed to propagate at a greater rate [5]. Therefore, the improvement of membrane brittleness and stimulate bone tissue formation is highly desired for the function and ultimate clinical application of GTR membrane.

However, CS/PEG membrane obtained through blending process has a poor antiacid ability and therefore needs to be cross-linked. The common used cross-linking reagents such as glutaraldehyde, epichlorohydrin, and cycloheptamylose are not efficient because they can make the membrane very brittle [9]. They also may lead to a toxic effect in physiological environment, due to the presence of residual crosslinker [10]. Naturally occurring cross-linker, such as reduced native mono- (i.e., glucose and fructose) and nonreduced disaccharides (i.e., sucrose), has reported in our group that the aldehyde or keto group of reduced sugar can react with the free amino groups of CS resulting in a partially soluble Schiff base products in low $\mathrm{pH}$ environment [11]. Therefore, fructose solution was prepared as cross-linking solution in this study. In addition, the Schiff base by-products (furan) in the composite only liberate in the first few days and the releasing amount is low. As a result, it may have a little adverse effect on cell growth during soaking [12].

It has been reported that natural or synthetic polymers alone may not be able to meet all the requirements of the budding bone cells [13]. To increase the bone affinity of CS/PEG membranes, the addition of other components for enhancing tissue regeneration has been investigated, such as growth factors, drugs, or bioceramics [14]. Bioceramics such as hydroxyapatite and $\beta$-tricalcium phosphates are chemically stable and compositionally similar to the mineral phase of the bone. In addition, bioceramics provide mechanical strength to the natural/synthetic biopolymers used for developing barrier membranes. Consequently, to use the bioresorbable ceramic as fillers for CS/PEG polyblend would have distinct advantages, not only they may reinforce polyblend structure but also they can facilitate the rate of integration of implant with bone and be resorbed by human body finally.

$\beta$-Tricalcium phosphate ( $\beta$-whitlockite) has excellent biocompatibility, bone bonding, and bioresorption abilities as a promising biofiller [15]. $\beta$-TCP ceramics can be gradually absorbed which followed by new bone formation yet without compromising the intimacy of bone-implant contact [16]. When osteoblasts are guided by $\mathrm{Ca}^{2+}$ and $\mathrm{PO}_{4}{ }^{3-}$ released from $\beta$-TCP, the bone cells would be directed to mineralization. Therefore, it is considered as an ideal material which can be added to the CS/PEG membranes for enhancing bone tissue regeneration.

We previously reported that the CS/PEG/ $\beta$-DCP membranes prepared by thermally induced phase separation method and following treatment with nontoxic glucose cross-linking agents exhibited similar properties with CS membrane that was strengthened by glutaraldehyde as crosslinking agent [12]. In this study, a mixture of $\beta$-TCP powder and fructose mediated CS/PEG membrane, denoted as $\mathrm{F}-\beta$-TCP/CS/PEG composite membrane, was prepared. Some basic properties of the F- $\beta$-TCP/CS/PEG composite membrane were examined, such as swelling measurements, mechanical strength, and degradation tests. The NIH3T3 fibroblast was prepared and cultured for testing the cytotoxicity of the F-CS/PEG/ $\beta$-TCP composite membrane.

\section{Materials and Methods}

2.1. Materials. CS with a high molecular weight (degree of deacetylation is $83 \%, \mathrm{Mw} \sim 4 \times 10^{5}$ ) was obtained in the form of flake from Fluka (Buchs, Switzerland). PEG $(\mathrm{Mn}=6000)$ was purchased from Riedel-de Haën (Seelze, Germany); fructose anhydrous was used as cross-linking agent and supplied by J. T. Baker Inc. (Phillipsburgh, USA). Tricalcium phosphate $(\beta$-TCP), purchased from Merck-Schuchardt (Germany), was screened through a 120 mesh sieve. All other reagents were of extra pure grade and were used as received.

\subsection{Preparation of Fructose Mediated $\beta-T C P / C S / P E G$ Com-} posite Membrane. CS (3 g) was dissolved in $100 \mathrm{~mL}$ of $2 \%$ aqueous acetic solution. A CS/PEG blend solution was prepared by the mechanical stirring of the CS and PEG flakes in a ratio of $70: 30$ at room temperature. Then $\beta$-TCP granules with $20,40,50,60$, and $75 \mathrm{wt} \%$ were added into the prepared solution and then stirred for $24 \mathrm{hr}$. The $5 \mathrm{wt} \%$ fructose was then added into the mixture and the final $\mathrm{pH}$ value of fructose-mediated CS/PEG gel was adjusted to 6 by $1 \mathrm{M}$ sodium hydroxide [17]. Then the fructose-mediated $\beta$-TCP/CS/PEG gel was thermally treated at $120^{\circ} \mathrm{C}$ in an oven for $1 \mathrm{~h}$. The membranes of the resulting polymer blend were obtained by casting into polystyrene Petri dishes and incubating for 2 day in an oven at $68^{\circ} \mathrm{C}$. The membrane was rinsed with deionized distilled water (DDW) and dried at ambient temperature overnight.

2.3. Mechanical Analysis. Tensile strength and elongation at break of the films were measured on a SANS CMT-6503 test machine versatile tester (Shenzhen, China) according to the ISO6239-1986 standard with a tensile rate of $5 \mathrm{~mm} \mathrm{~min}^{-1}$. The size of the films was $70 \mathrm{~mm}$ length, $10 \mathrm{~mm}$ width, with $50 \mathrm{~mm}$ distance between two clamps. Five parallel measurements were carried out for every sample.

2.4. Swelling Behavior of Composite Membranes. The membrane was dried first in a vacuum oven at $50^{\circ} \mathrm{C}$ until it reached a constant weight. The swelling behavior of the membrane was determined by swelling the membrane $\left(1 \times 1 \mathrm{~cm}^{2}\right)$ in $\mathrm{pH} 7.4$ of phosphate-buffered saline (PBS) at $37^{\circ} \mathrm{C}$. After 
a given time interval (1-2 hr), the excess surface solution was blotted out with filter paper. The swelling procedure was repeated until there was no further weight increase. The swelling degree for each sample at time $t$ was calculated using the relation $\left(W_{t}-W_{0}\right) / W_{0}$, where $W_{t}$ is the weights of the swollen membrane at time $t$ and $W_{0}$ is the initial weight of the membrane. The measurement was repeated several times to obtain an average value of swelling degree for each sample.

2.5. In Vitro Degradation Test. The composite was cut into a 1 $\times 1 \mathrm{~cm}^{2}$ film with $0.2 \mathrm{~g}$ in weight and placed in a $20 \mathrm{~mL}$ scintillation vial with $4 \mathrm{mg} \mathrm{mL}^{-1}$ lysozyme in $\mathrm{pH} 7.4$ phosphate buffer solution (PBS) inside. All of the vials were kept in an incubator at a temperature of $37^{\circ} \mathrm{C}$. After soaking for $2,7,14$, 21 , and 28 days, respectively, the composite membranes were taken out of the immersion solution, and then the degradation profiles were expressed as the accumulated weight losses of the membrane. The microstructure and morphology of the crystals deposited on the surface were observed under SEM (JEOL 5120). The crystalline phases of specimens were determined by Rigaku X-ray powder diffractometry with $\mathrm{Cu}$ $\mathrm{K}_{\alpha}$ radiation and $\mathrm{Ni}$ filter. The scanning range of the samples was from $5^{\circ}$ to $50^{\circ}$ at a scanning speed of $4^{\circ} / \mathrm{min}$ with the accelerating voltage of $30 \mathrm{KV}$ and current of $20 \mathrm{~mA}$. The extracts from composite membrane were collected for use in cell culture and examined by gas chromatography-mass spectrometry (GC-MAS) and inductively coupled plasma atomic emission spectrometry (ICP-AES).

2.6. Analysis of Extracts from Composite Membrane. In order to measure the content of constituents within extracts that have been released in the series from the composite membrane, composite membrane was soaked within the PBS solution along with lysozyme for some time. The calcium and phosphate contents in the extracts were determined by ICP-AES. The GC-MAS was used to detect the amount of glucose amine and Schiff base by-products. GC-MS analyses were performed using Agilent 5973 network mass selective detectors fitted with an Agilent 6890N GC gas chromatograph and an on-column injector, using helium as the carrier gas. Extracts were separated on a HP-5MS column $(0.25 \mathrm{~mm}$ $\times 30 \mathrm{~m}$ (id), $0.25 \mu \mathrm{m}$ film thickness). The GC oven was operated as follows: isothermal for $2 \mathrm{~min}$ at $50^{\circ} \mathrm{C}$; temperature programmed at $10^{\circ} \mathrm{C} / \mathrm{min}$ to $225^{\circ} \mathrm{C}$; and then isothermal for $15 \mathrm{~min}$. The MS was operated in full scan mode (47$400 \mathrm{au}, 3$ scans/sec). Peaks were identified based on their mass spectral characteristics and GC retention indices, by comparison with commercial glucosamine (Aldrich, U.K.).

2.7. Cytotoxicity Assay. To determine the influence of composite membrane extracts on the growth and morphological transformation of the NIH3T3 fibroblast, the extracts for use in cell cultures were sterilized by filtrating through $0.45 \mu \mathrm{m}$ millipore filters and adding to cell cultures.

The NIH3T3 fibroblasts were cultured in Dulbecco's modified Eagle's medium (DMEM; Gibco, USA) supplemented with $10 \%$ fetal bovine serum (FBS; Gibco, USA) and $1 \%$ antibiotic-antimycotic (Gibco, USA). The cells were subcultured for about 3-day interval with trypsin-EDTA and maintained at $37^{\circ} \mathrm{C}$ in a water-jacked incubator with a humidified $5 \% \mathrm{CO}_{2}$ atmosphere. The fibroblasts were placed in a six-welled tissue culture plate $\left(1 \times 10^{5}\right.$ cells per well $)$ in a complete medium and incubated for $24 \mathrm{hr}$. The medium was then replaced and the extracts from the series of fructose mediated $\beta$-TCP composite membrane were added in a ratio of $1: 1$ for the medium and extract, giving a final extract concentration of $50 \%$. In the control group, the phosphate buffer solution (PBS) with lysozyme was mixed with the complete medium in a ratio of $1: 1$ for cell culture. After being cocultured for 2 days, the medium was removed and the cells were trypsinized and resuspended, and then $1 \mathrm{~mL}$ trypan blue was added to make sure that the cells were still living and then they were counted in a Neubauer counting-chamber under an optical microscope.

\section{Results and Discussion}

3.1. Morphology of F- $\beta$-TCP/CS/PEG Membrane. Figure 1 shows the morphologies of F-CS/PEG membrane and FCS/PEG membrane with different $\beta$-TCP feeding weight. The F-CS/PEG membrane shows a dense and compact surface pattern. It may be due to the keto group of fructose reactive with free amino group of CS made the CS main chain rigid and restricted its engagement at high $\mathrm{pH}$ mediating environment [11]. With the $\beta$-TCP content increased from $20 \mathrm{wt} \%$ to $75 \mathrm{wt} \%$ (Figures $1(\mathrm{~b})-1(\mathrm{~d})$ ), the surface roughness of F-CS/PEG membrane increased obviously due to the separation of $\beta$-TCP crystal dispersed throughout F-CS/PEG membrane. Furthermore, by increasing the fraction of $\beta$ TCP more than $50 \mathrm{wt} \%$, a somewhat aggregation of particles was yielded within the membrane, yet resulting in the discontinuous structure of membrane. This discontinuous structure of composite membrane was disadvantage for the further in vivo application [18]. Figure 2 also shows the XRD patterns of F-CS/PEG composite membranes with different $\beta$-TCP feeding weight. All characteristic peaks of the patterns are in agreement with the X-ray diffracted JCPD data files of $\beta$-TCP and CS. The peak found at $20^{\circ}$ is assigned to CS chain aligned through intermolecular interactions. The composite can be described to the $\beta$-TCP structure with the peaks detected at $31.7^{\circ}$ for (211), $34.1^{\circ}$ for (202), and $25.9^{\circ}$ for (002). It was found that the crystallinity of CS was decreased with increasing $\beta$-TCP content, which is probably caused by the well-dispersed $\beta$-TCP particles which act as point defects in the CS/PEG matrix [19]. In addition, the strong polymerfiller interaction could change the molecular conformation of polymer chain in the vicinity of filler and simultaneously gave rise to the formation of localized amorphous regions. This retardation becomes more pronounced when the $\beta$-TCP content exceeds $20 \mathrm{wt} \%$ [20].

3.2. Mechanical Properties. As a GTR barrier material, the $\mathrm{F}-\beta$-TCP/CS/PEG membrane required adequate mechanical strength. Moreover, the appropriate degradation rate of material also has to fit the requirement of tissue regeneration. To investigate the effect of $\beta$-TCP content on the mechanical 


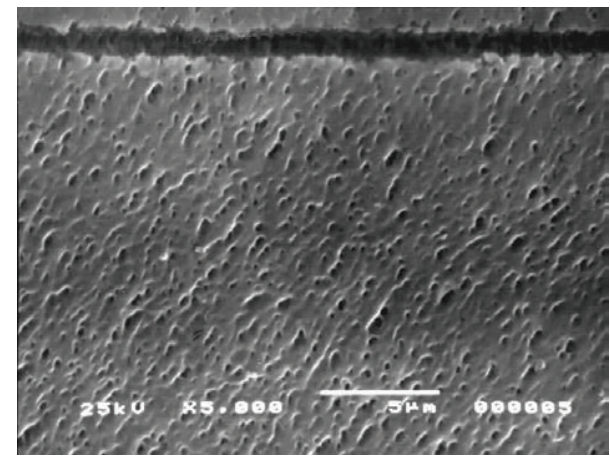

(a)

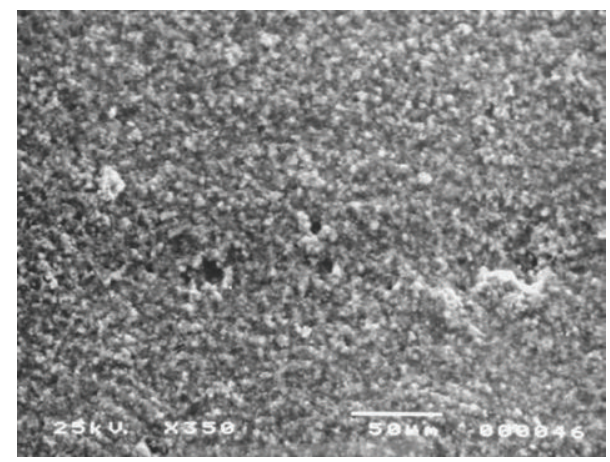

(c)

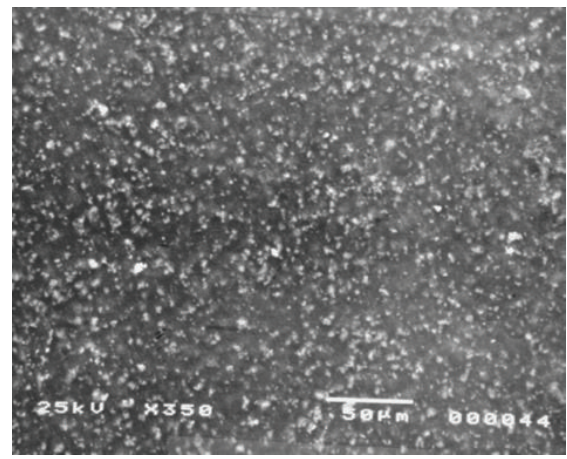

(b)

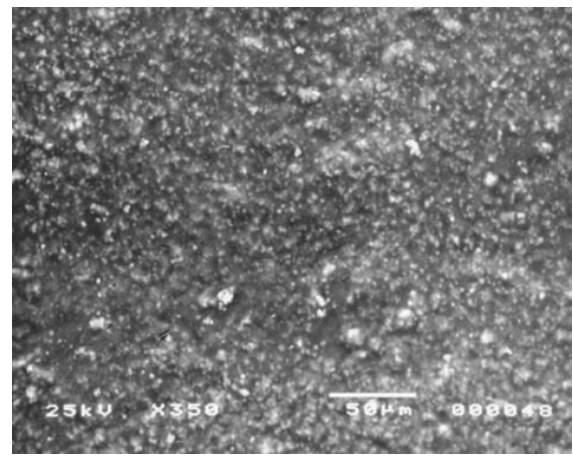

(d)

FIGURE 1: Surface morphologies of F-CS/PEG membrane and F-CS/PEG membrane with different $\beta$-TCP feeding weight (a) F-CS/PEG; (b) $20 \mathrm{wt} \% \beta$-TCP; (c) $50 \mathrm{wt} \% \beta$-TCP, and (d) $75 \mathrm{wt} \% \beta$-TCP.

property of the F-CS/PEG composite membranes, tensile test were performed. Figure 3 shows the tensile strength ( $\mathrm{sb})$ and elongation at break (eb) of the composite membrane. It revealed that the tensile strength of composite membrane showed great dependence on the $\beta$-TCP content. The elongation at break of the composite membrane decrease from $6.14 \%$ to $1.76 \%$ as the $\beta$-TCP content increased. The tensile strength increased from $21.54 \pm 1.71 \mathrm{MPa}$ to $58.14 \pm$ $4.65 \mathrm{MPa}$ as the $\beta$-TCP content increasing from $0 \mathrm{wt} \%$ to $50 \mathrm{wt} \%$. Following the $\beta$-TCP content increase from $50 \mathrm{wt} \%$ to $75 \mathrm{wt} \%$, the tensile strength decreased dramatically from $58.14 \pm 4.65 \mathrm{MPa}$ to $18.27 \pm 1.47 \mathrm{MPa}$. According to $\mathrm{Li}$ et al's [18] study, $\beta$-TCP particles could disperse homogeneously in the F-CS/PEG solution and did not separate from the membrane during the fabrication process as the $\beta$-TCP content was lower than $50 \mathrm{wt} \%$. However, following the $\beta$ TCP content higher than $50 \mathrm{wt} \%$, the agglomeration of $\beta$-TCP particles resulted in the discontinuous structure of membrane (as seen in Figure 1(c)). Therefore, the tensile strength of composite membrane decreased dramatically as the $\beta$-TCP content was greater than $50 \mathrm{wt} \%$. The results indicate that the $\beta$-TCP content must be controlled to be lower than $50 \mathrm{wt} \%$ to obtain homogeneous F-CS/PEG membrane with well mechanical property.

3.3. Swelling Study. In clinical practice, when materials are used as tissue regeneration barrier membranes, the rapid swelling phenomenon would be beneficial to the clinical manageability and surgical procedures [21]. Figure 4 shows the behavior of swelling degree of composite membrane. All membranes could reach steady hydration equilibrium state within the $30 \mathrm{~min}$ period of the experiment. The swelling degree of the F-CS/PEG reaches the highest point of $68 \%$ after $1 \mathrm{~h}$. However, following the $\beta$-TCP content increased results in a significant reduction in swelling degree of composite. The swelling degree of composite is reduced when incorporating $\beta$-TCP with F-CS/PEG matrix due to formation of a temporary $\beta$-TCP barrier preventing water permeating into F-CS/PEG, which postponed the attenuation of mechanical properties of composite under moisture condition.

3.4. Degradation Behavior In Vitro. As a guided regeneration barrier material, the appropriate degradation rate of material ought to be managed to fit into the schedule of remodeling of tissue regeneration [22]. To estimate the biodegradability behavior of the composite membrane, the extent of degradation of the F-CS/PEG and composite membranes after 28 days of immersion in PBS solution containing lysozyme enzyme $(4 \mathrm{mg} / \mathrm{mL})$ is shown in Figure 5. All membranes were degradable, and the composite membranes had lower weight loss than the F-CS/PEG membranes. The weight loss of the composite membranes decreases as the $\beta$-TCP content increased. The F-CS/PEG is susceptible to hydrolysis by lysozyme at $\mathrm{pH} 7.4 \mathrm{PBS}$ solution and the weight loss reaches $28 \%$ after soaking for 7 days. However, the bulk form of $\beta$-TCP degradation rate is slow compared to F-CS/PEG membrane [2]. It indicated that the $\beta$-TCP particles in the F-CS/PEG matrix could affect the binding of enzymes on 


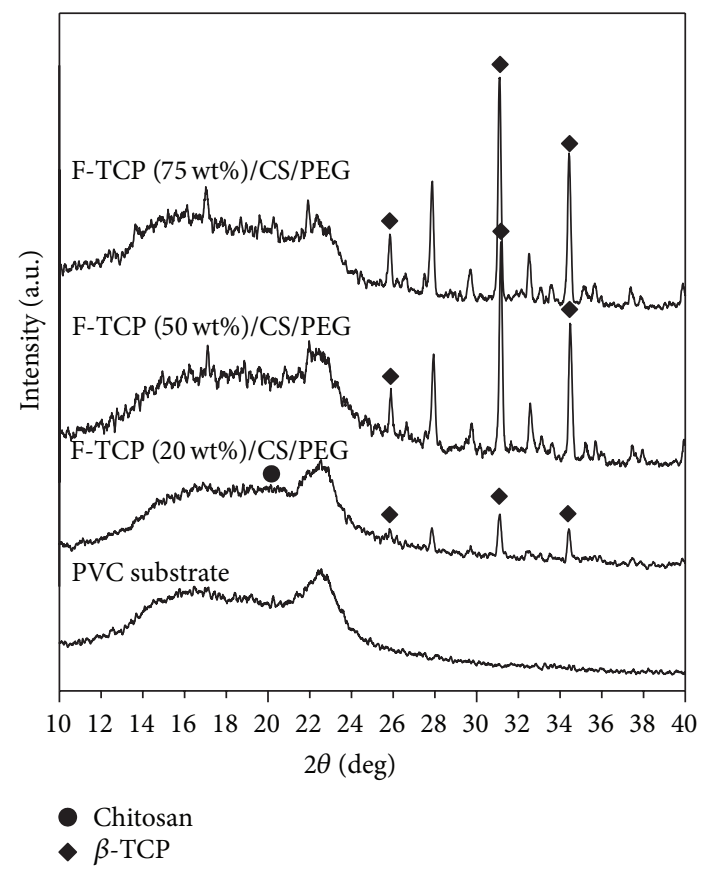

FIGURE 2: XRD patterns of F-CS/PEG composite membranes with different $\beta$-TCP feeding weight.

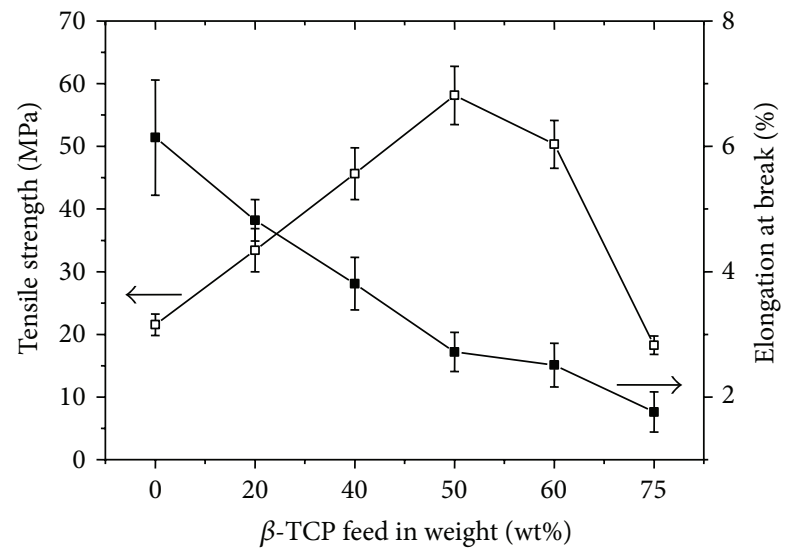

Figure 3: Tensile strength (sb) and elongation at break (eb) of F-CS/PEG composite membranes with different $\beta$-TCP feeding weight.

the F-CS/PEG, thereby decreasing the degradability [12]. For low $\beta$-TCP feeding weight (20 wt $\%$ and $40 \mathrm{wt} \%$ ) of the $\mathrm{F}$ CS/PEG composite, the main degradation mechanism may be mainly controlled by the F-CS/PEG degradation rate due to more reactive site for lysozyme attacking. But as the $\beta$-TCP content is higher than $40 \mathrm{wt} \%$, the site for lysozyme attacking decreases. Therefore, the high $\beta$-TCP feeding weight of the F-CS/PEG composite degradation behavior is mainly controlled by the slow degradation rate of $\beta$-TCP particle.

Figure 6 shows the morphology of $50 \mathrm{wt} \% \mathrm{~F}-\beta$-TCP/CS/ PEG composite membranes immerged in the PBS solution containing lysozyme enzyme for 28 days. The initial $50 \mathrm{wt} \% \mathrm{~F}-\beta$-TCP/CS/PEG membrane exhibits a rough surface (Figure 6(a)). When immerged in PBS for 7 days, the

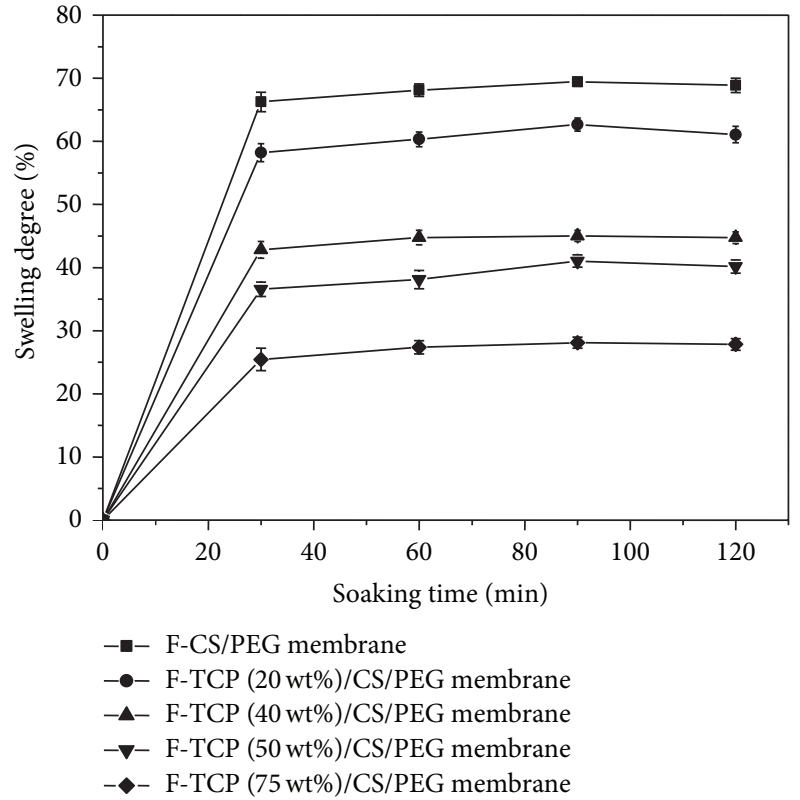

FIGURE 4: Swelling behavior of F-CS/PEG composite membranes with different $\beta$-TCP feeding weight.

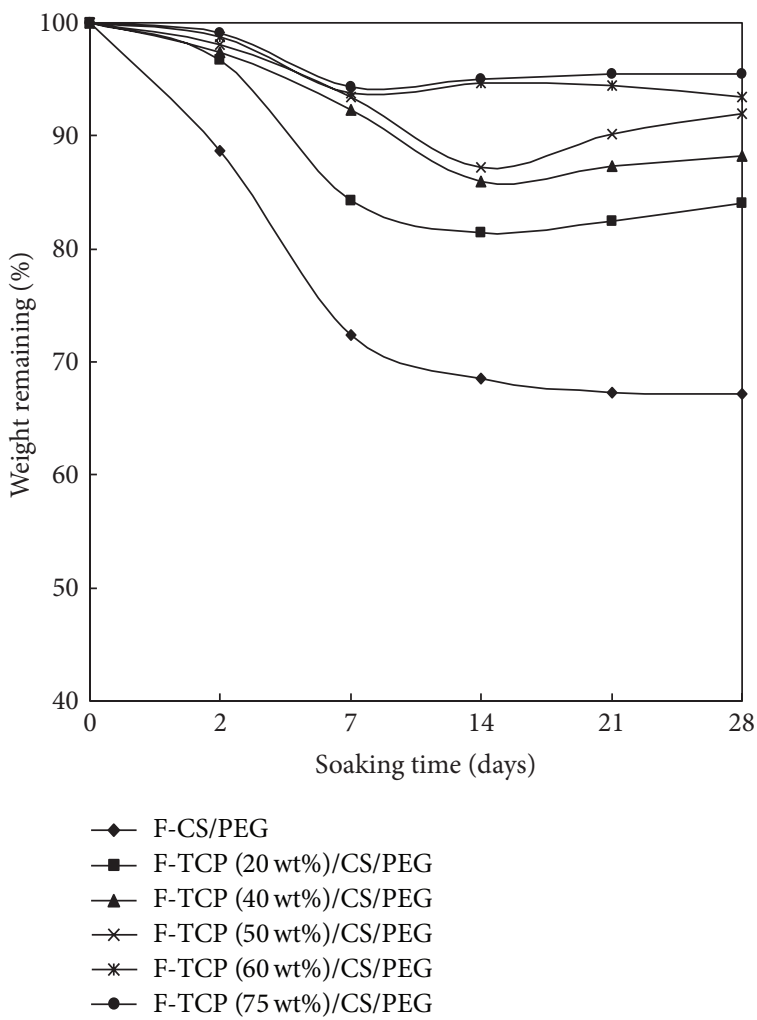

FIgURE 5: Weight loss curves of the series of F- $\beta$-TCP/CS/PEG composite soaking in a PBS solution containing lysozyme enzyme for 28 days. 


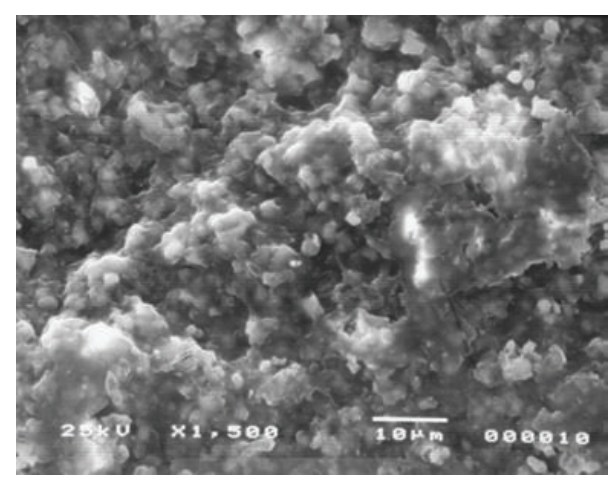

(a)

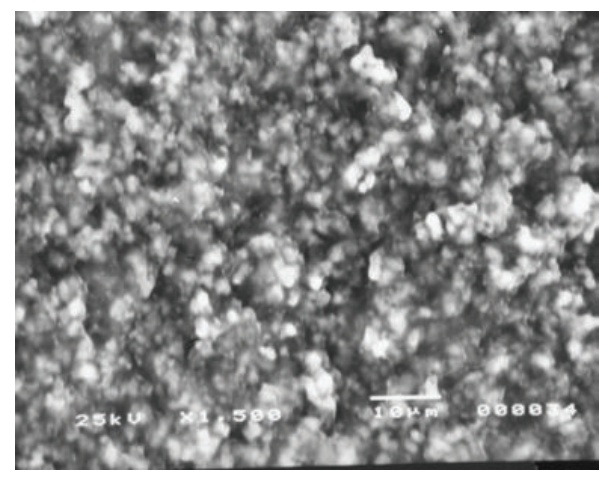

(c)

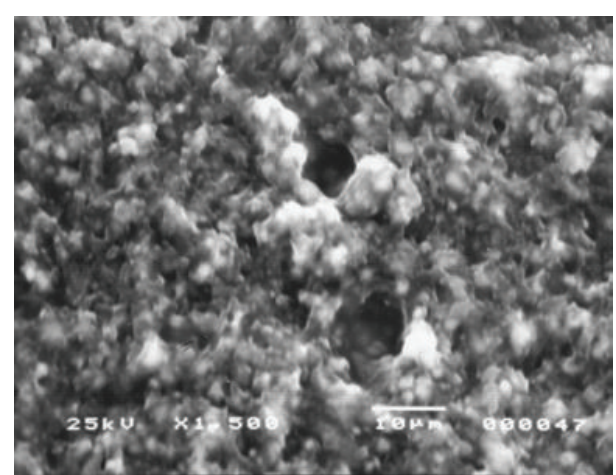

(b)

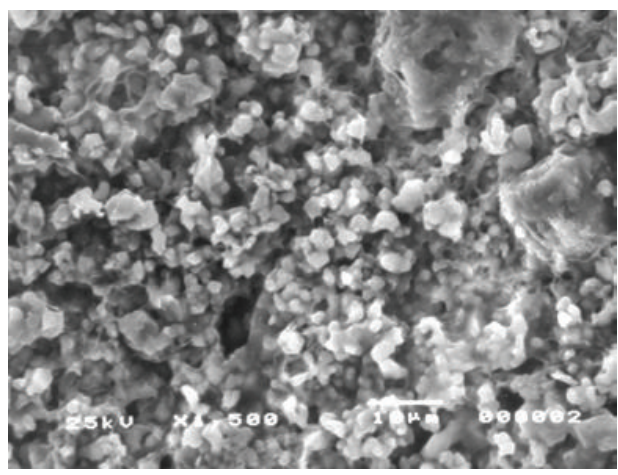

(d)

FIGURE 6: Surface morphology of $50 \mathrm{wt} \%$ F- $\beta$-TCP/CS/PEG composite membranes immerged in the PBS solution containing lysozyme enzyme for 28 days. (a) 0 days; (b) 7 days; (c) 14 days; and (d) 28 days.

surface of $50 \mathrm{wt} \% \mathrm{~F}-\beta$-TCP/CS/PEG is partly dissolved and appears to have round pores on the surface (Figure 6(b)). F-CS/PEG of the membrane surface behaves preferential hydrolytic scission into low molecules, which can dissolve in PBS. Figure 6(c) indicates that some round voids and degraded fragment spread over the sample's surface after 14 days. It is coincident with the result of $50 \mathrm{wt} \% \mathrm{~F}-\beta$ TCP/CS/PEG weight loss during the 7-14 days. With the prolongation of the degradation time, a lot of concaves appeared on the film surface because of the further erosion by PBS. Figure 6(d) shows that lots of $\beta$-TCP particles are exposed on the surface. It indicates that the outer surface of F-CS/PEG matrix is dissolved after immersing for 28 days. In addition, many concaves are presented on the surface and the composite structure starts to disintegrate due to the degradation of F-CS/PEG and the $\beta$-TCP decomposes to calcium ion and phosphate ion. The phenomenon can be proved by analyzing the degradation products from composite membrane extracts. The extracts contain lots of degradation products, including low molecular chitosan, glucosamine, Schiff base product, calcium ion, and phosphate ion [12]. Figure 7 shows the concentration of glucosamine molecules in the extracts of series of composites soaked in a PBS solution containing lysozyme. It demonstrates that the glucosamine release decreased as $\beta$-TCP content increased in the composite. The low $\beta$-TCP content composite membrane has the similar degradation behavior of F-CS/PEG membrane. However, when the $\beta$-TCP content is higher than
$50 \mathrm{wt} \%$, the glucosamine molecules releasing rate was slow down. It may be due to the high content $\beta$-TCP particles shielding the F-CS/PEG cross-linked structure.

Figure 8 shows the calcium ion concentration after the F$\beta$-TCP/CS/PEG composites are immersed in a PBS solution containing lysozyme enzyme for 2-28 days. It demonstrates that the calcium ion release slightly increased in the initial 2 days as $\beta$-TCP content increased in the composite. It can be inferred that the more the $\beta$-TCP particles on the free surface of the composite membrane, the more the calcium ions release in the extracts. After 7 days, the F-CS/PEG decomposes and is gradually released from composite and then $\beta$-TCP particles are continuously exposed and dissolved in the PBS solution, which would explain the continuously increasing calcium ion concentrations in the extracts. This also can be verified by the glucosamine releasing profile. However, as the $\beta$-TCP content increases to $75 \mathrm{wt} \%$, the calcium ion releasing rate decreases to be slower than $50 \mathrm{wt} \% \mathrm{~F}-$ $\beta$-TCP-CS/PEG after 14 days. Due to the physical interlocking between $\beta$-TCP and F-CS/PEG particles, the undissolved $\beta$ TCP retards the degradation of F-CS/PEG, which makes a lower calcium ion releasing rate in the period of 7 to 14 days. In addition, as the $\beta$-TCP content increases to $75 \mathrm{wt} \%$, aggregations of particles were presented in the membrane, which will prolong the dissolving process in the PBS solution. Therefore, the degradation rate is slow for $75 \mathrm{wt} \% \mathrm{~F}-\beta$-TCPCS/PEG composite membrane. 


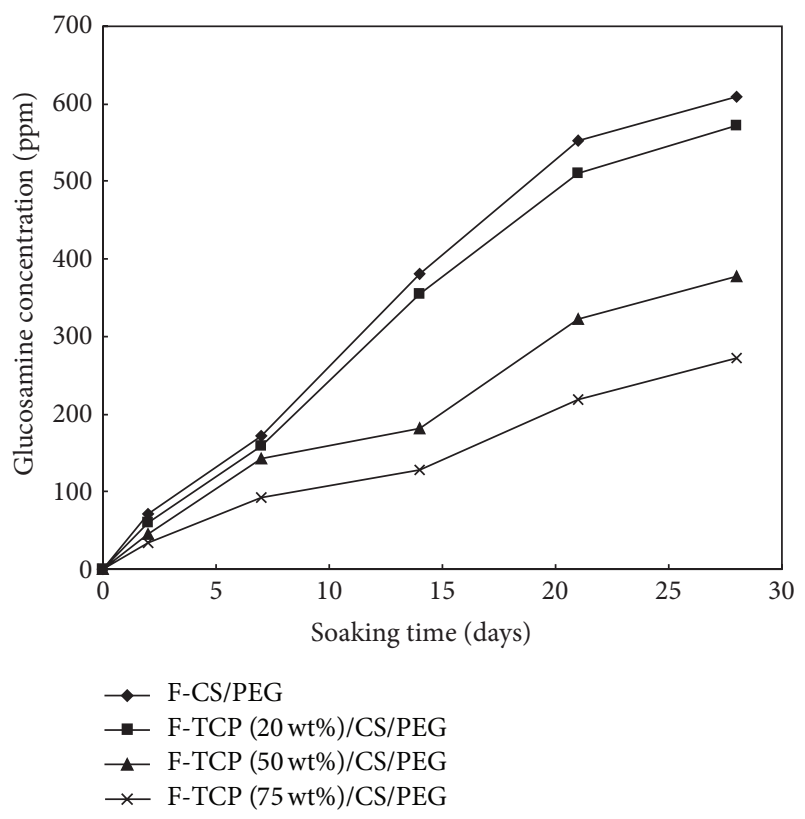

FIGURE 7: Relationship between glucosamine in the extraction and soaking periods of the series of $\mathrm{F}-\beta$-TCP/CS/PEG composite in a PBS solution containing lysozyme enzyme.

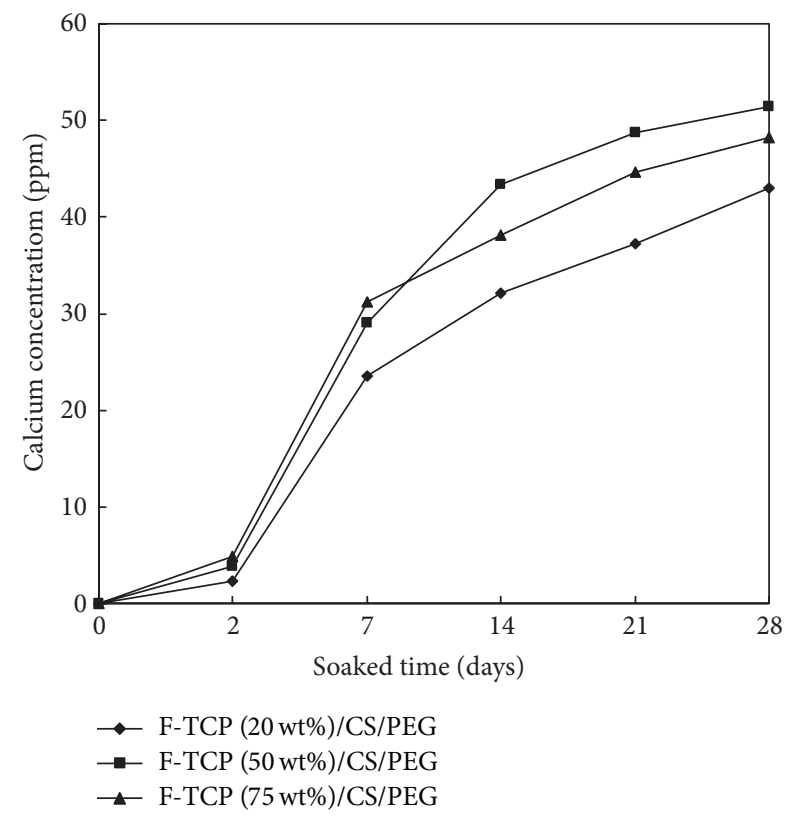

FIgURE 8: Relationship between calcium content in the extracts and soaking periods of the series of $\mathrm{F}-\beta$-TCP/CS/PEG composite in a PBS solution containing lysozyme enzyme.

Figures 9 and 10 show the XRD patterns of $20 \mathrm{wt} \%$ and $50 \mathrm{wt} \% \mathrm{~F}-\beta$-TCP/CS/PEG composite membranes after immersion in PBS containing lysozyme enzyme for 28 days. There is no phase transformation or reaction occurring and no other new phase appears during the immersion period. For $20 \mathrm{wt} \% \mathrm{~F}-\beta$-TCP/CS/PEG composite, the characteristic peak of F-CS/PEG around $20^{\circ}$ becomes broaden after 14

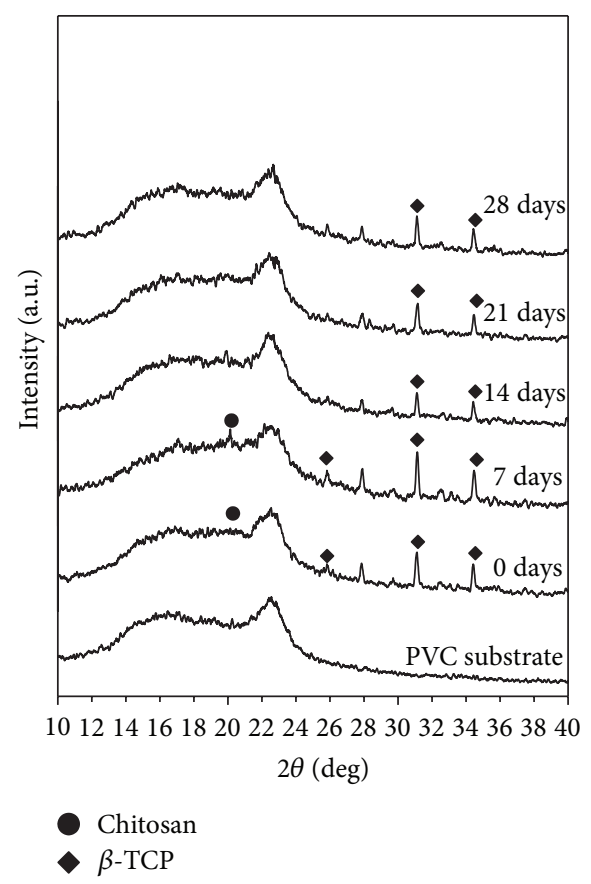

FIGURE 9: XRD patterns of $20 \mathrm{wt} \% \mathrm{~F}-\beta$-TCP/CS/PEG composite after immersion in a PBS solution containing lysozyme enzyme for 0-28 days.

days. It indicated that the crystal structure of F-CS/PEG is distorted after 14 days immersion time. In addition, all characteristic peaks of the $\beta$-TCP gradually decrease as the immersion time increased. It indicates that phase TCP could be dissolved in PBS solution, leading to TCP disintegration. The intensity of $\beta$-TCP peaks is not an obvious change after 14 days immersion time as $\beta$-TCP content increased to $50 \mathrm{wt} \%$. It demonstrates that the $\beta$-TCP retards the degradation of F-CS/PEG during 7-14 days resulting in a slight increase in the calcium ion concentration. But the intensity of $\beta$-TCP peaks was sharply decreased after 21 days, which inferred that $\beta$-TCP degradation rate increases resulting in the calcium concentration increasing after 21 days.

\subsection{Effects of F- $\beta$-TCP/CS/PEG Composite Membrane Extracts} on NIH3T3 Cell Culture. Figure 11 shows the cell numbers after culturing the NIH3T3 fibroblasts with extraction media of F- $\beta$-TCP/CS/PEG composite membranes for 4 different incubation time intervals for each sample $(2,7,14$, and 28 days). The cell numbers were significantly lower than that of control for composite membranes in the initial 2 days. Thereafter the cell numbers are higher than those of the control group for composite after 7 days. In addition, the cell numbers are not decreased by increasing incubation time of composite membrane. The Maillard reaction of chitosan with fructose takes place during the processing [17]. By further reaction, the strecker degradation would occur and form heterocyclic compounds such as furan, pyridine, pyrrole, and oxazole. It has been reported that heterocyclic compounds may show modest genotoxic and cytotoxic effects in high doses after long incubation times. However, the low 


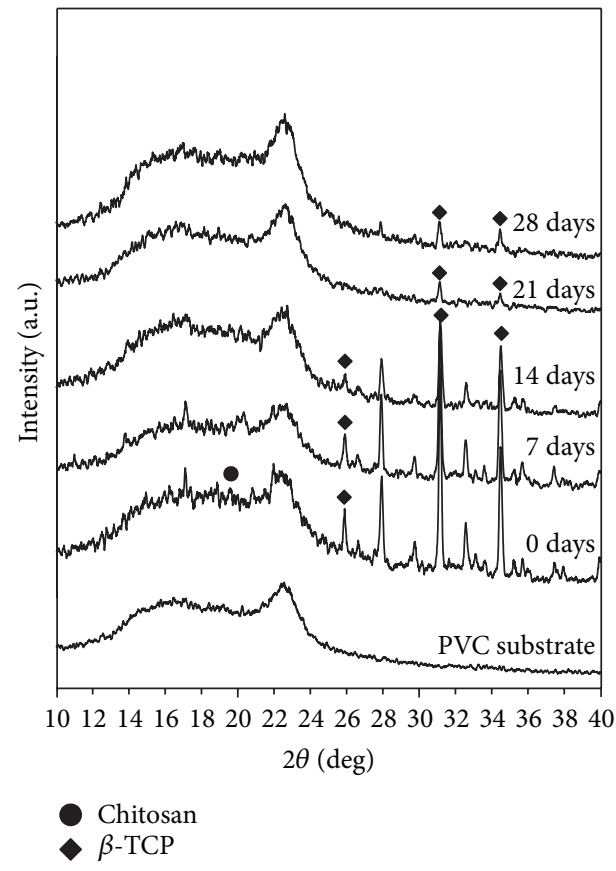

FIGURE 10: XRD patterns of $50 \mathrm{wt} \% \mathrm{~F}-\beta$-TCP/CS/PEG composite after immersion in a PBS solution containing lysozyme enzyme for $0-28$ days.

concentrations found in products do not pose a health risk [23]. Figure 12 shows the furan compound in the extracts of $20 \mathrm{wt} \% \mathrm{~F}-\beta$-TCP/CS/PEG composites soaked in a PBS solution containing lysozyme. Most of the furan compound releases within the first two days and after that reaches the plateau following soaking for seven days. It demonstrates that the residual furans in the $\mathrm{F}-\beta$-TCP/CS/PEG composite only liberate in the first few days and after that no more toxic agents were released by increasing incubation time. Therefore, $\beta$-TCP/CS/PEG composite membrane may have a little adverse effect on cell growth in the first few days.

Interestingly, the cell numbers are obviously higher than those of the control group for composite after 7 days. The degradation products including glucosamine and calcium ion have proved that is favorable to the growth of the myoblast. It has been proved that the glucosamine with concentration higher than $50 \mathrm{ppm}$ is a nutritious element for the fibroblast growth [24]. When the F- $\beta$-TCP/CS/PEG composites are soaked in the PBS solution containing lysozyme after 7 days, the glucosamine concentrations in the extracts are higher than $50 \mathrm{ppm}$ as shown in Figure 7. Therefore, the cell number exhibits an increasing tendency as the soaking time is over 7 days. In addition, the cell numbers increase as the $\beta$ TCP feed in weight increases. According to Barradas et al.s study [25], $\beta$-TCP composites were soluble in PBS solution and $\mathrm{Ca}^{2+}$ and $\mathrm{PO}_{4}{ }^{3-}$ are continuously released from the ceramic. In the present study, calcium ion is continuously increasing after 7 days as shown in Figure 8. Therefore, the cell numbers apparently increase with the calcium content increase. This proves the positive effect of the addition of $\beta$-TCP particles on cell proliferation. As a result, the F- $\beta$-TCP/CS/PEG composites membrane possesses excellent

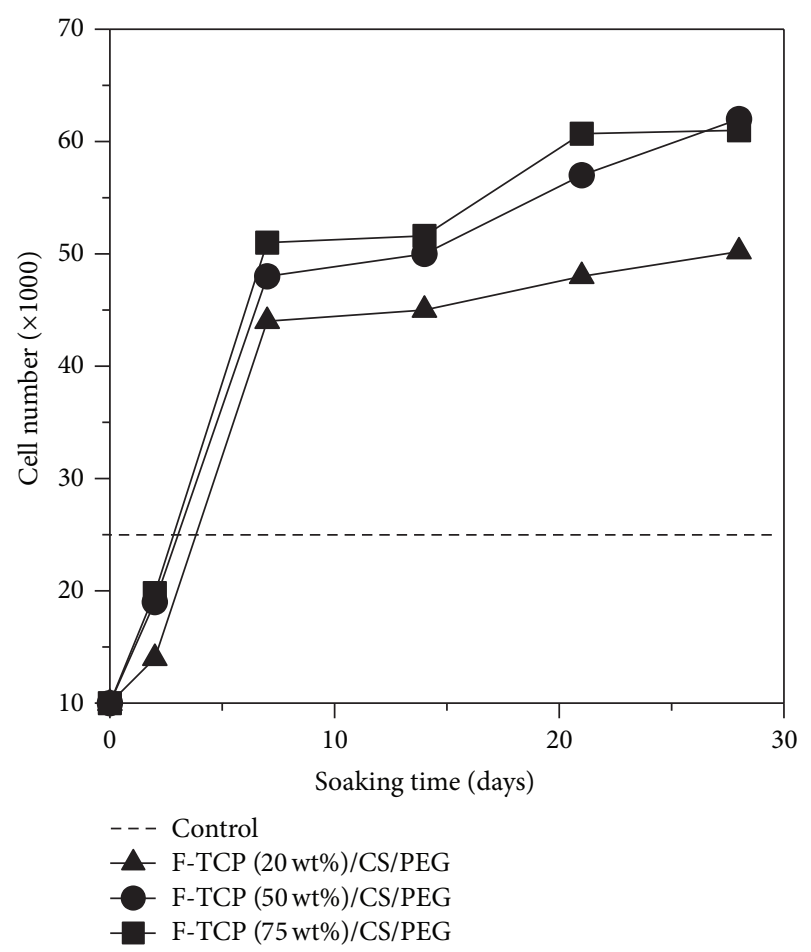

FIGURE 11: Relationship between cell number and extractive periods as the series of F- $\beta$-TCP/CS/PEG composite extract cocultured with NIH3T3 fibroblast for 2 days.

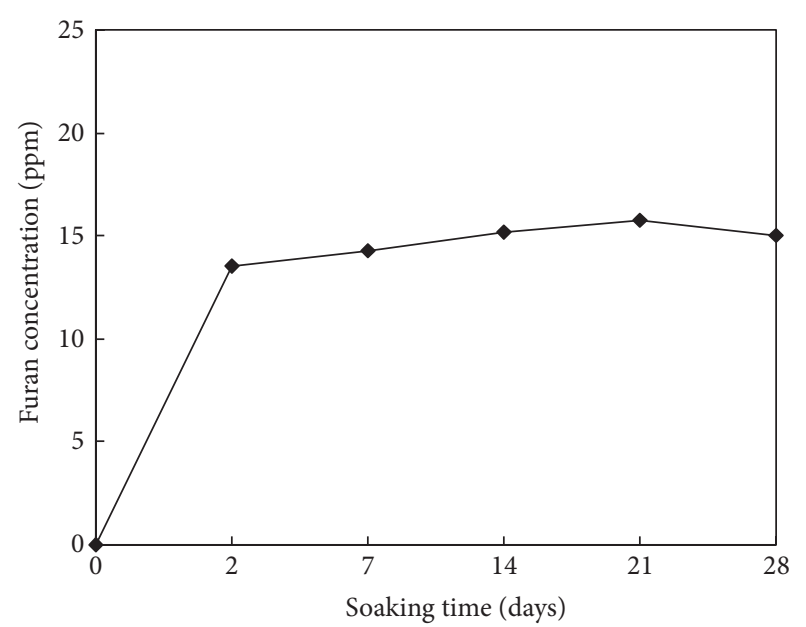

FIGURE 12: Relationship between furan in the extracts and soaking periods of the F- $\beta$-TCP/CS/PEG composite in a PBS solution containing lysozyme enzyme.

biocompatible and biodegradable characteristics which met the requirements of barrier membrane used in GTR.

\section{Conclusion}

In the present study, F- $\beta$-TCP/CS/PEG composites membrane is developed as a barrier membrane used in GTR techniques. Results show that the $\beta$-TCP content could be controlled to modulate the structure of the composite 
membranes and in turn could cause different behaviors in water absorption, enzymatic degradability, and mechanical properties. The $\beta$-TCP particles incorporated into F-CS/PEG membrane could further increase the rigidity and decrease the degradability of the membranes. When $\beta$-TCP content is controlled to $50 \mathrm{wt} \%$, homogeneous composite membranes with well mechanical property and enzymatic degradation rate can be obtained. The composite membranes could reasonably meet the degradation requirement of bioresorbable membrane used for tissue regeneration. Cytotoxicity assay demonstrates that the composite membranes were nontoxic and had very good cell compatibility. Most importantly, the release of calcium ions and glucosamine from the composite membranes was proved to increase the cell proliferation of NIH3T3. The findings of this initial study have indicated that this novel F- $\beta$-TCP/CS/PEG composite is a potential candidate as the barrier membrane for GTR applications.

\section{Conflict of Interests}

The authors declare that there is no conflict of interests regarding the publication of this paper.

\section{Acknowledgment}

The authors gratefully acknowledge the financial support from the National Science Council of Taiwan (Grant no. 1012221-E-273-003-MY2).

\section{References}

[1] T. W. Chen, S. J. Chang, G. C.-C. Niu, Y. T. Hsu, and S. M. Kuo, "Alginate-coated chitosan membrane for guided tissue regeneration," Journal of Applied Polymer Science, vol. 102, no. 5, pp. 4528-4534, 2006.

[2] S. M. Kuo, S. J. Chang, G. C.-C. Niu, C.-W. Lan, W. T. Cheng, and C. Z. Yang, "Guided tissue regeneration with use of B-TCP/chitosan composite membrane," Journal of Applied Polymer Science, vol. 112, no. 5, pp. 3127-3134, 2009.

[3] K. Zhang, M. Zhao, L. Cai, Z.-K. Wang, Y.-F. Sun, and Q.-L. $\mathrm{Hu}$, "Preparation of chitosan/hydroxyapatite guided membrane used for periodontal tissue regeneration," Chinese Journal of Polymer Science, vol. 28, no. 4, pp. 555-561, 2010.

[4] H. Tan, R. Ma, C. Lin, Z. Liu, and T. Tang, "Quaternized chitosan as an antimicrobial agent: antimicrobial activity, mechanism of action and biomedical applications in orthopedics," International Journal of Molecular Sciences, vol. 14, no. 1, pp. 1854-1869, 2013.

[5] H. Hong, C. Liu, and W. Wu, "Preparation and characterization of chitosan/PEG/gelatin composites for tissue engineering," Journal of Applied Polymer Science, vol. 114, no. 2, pp. 1220-1225, 2009.

[6] J.-W. Wang and M.-H. Hon, "Effects of sugar cross-linking agents and thermal treatment on the culture of fibroblasts in vitro on a (PEG/chitosan) membrane," Journal of Biomaterials Science, Polymer Edition, vol. 14, no. 2, pp. 119-137, 2003.

[7] L.-H. He, R. Xue, D.-B. Yang, Y. Liu, and R. Song, "Effects of blending chitosan with peg on surface morphology, crystallization and thermal properties," Chinese Journal of Polymer Science, vol. 27, no. 4, pp. 501-510, 2009.
[8] A. P. Hurt, G. Getti, and N. J. Coleman, "Bioactivity and biocompatibility of a chitosan-tobermorite composite membrane for guided tissue regeneration," International Journal of Biological Macromolecules, vol. 64, pp. 11-16, 2014.

[9] M. Li, S. Cheng, and H. Yan, "Preparation of crosslinked chitosan/poly(vinyl alcohol) blend beads with high mechanical strength," Green Chemistry, vol. 9, no. 8, pp. 894-898, 2007.

[10] A. Altinisik and K. Yurdakoc, "Synthesis, characterization, and enzymatic degradation of chitosan/PEG hydrogel films," Journal of Applied Polymer Science, vol. 122, no. 3, pp. 1556-1563, 2011.

[11] J. W. Wang and M. H. Hon, "Preparation and characterization of $\mathrm{pH}$ sensitive sugar mediated (polyethylene glycol/chitosan) membrane," Journal of Materials Science: Materials in Medicine, vol. 14, no. 12, pp. 1079-1088, 2003.

[12] J. W. Wang and M. H. Hon, "Biodegradation behavior and cytotoxicity of the composite membrane composed of $\beta$ dicalcium pyrophosphate and glucose mediated (polyethylene glycol/chitosan)," Journal of Materials Science: Materials in Medicine, vol. 15, no. 2, pp. 129-136, 2004.

[13] B. L. Seal, T. C. Otero, and A. Panitch, "Polymeric biomaterials for tissue and organ regeneration," Materials Science and Engineering, R: Reports, vol. 34, no. 4-5, pp. 147-230, 2001.

[14] D. Mohamad Yunos, O. Bretcanu, and A. R. Boccaccini, "Polymer-bioceramic composites for tissue engineering scaffolds," Journal of Materials Science, vol. 43, no. 13, pp. 44334442, 2008.

[15] K. A. Hing, L. F. Wilson, and T. Buckland, "Comparative performance of three ceramic bone graft substitutes," The Spine Journal, vol. 7, no. 4, pp. 475-490, 2007.

[16] L. C. Lin, S. J. Chang, S. M. Kuo, G. C.-C. Niu, H. K. Keng, and P. H. Tsai, "Preparation and evaluation of $\beta$-TCP/polylactide microspheres as osteogenesis materials," Journal of Applied Polymer Science, vol. 108, no. 5, pp. 3210-3217, 2008.

[17] J.-W. Wang and Y.-M. Kuo, "Preparation of fructose-mediated (polyethylene glycol/ chitosan) membrane and adsorption of heavy metal ions," Journal of Applied Polymer Science, vol. 105, no. 3, pp. 1480-1489, 2007.

[18] X. Li, K. Nan, S. Shi, and H. Chen, "Preparation and characterization of nano-hydroxyapatite/chitosan cross-linking composite membrane intended for tissue engineering," International Journal of Biological Macromolecules, vol. 50, no. 1, pp. 43-49, 2012.

[19] T.-Y. Liu, S.-Y. Chen, J.-H. Li, and D.-M. Liu, "Study on drug release behaviour of CDHA/chitosan nanocomposites-effect of CDHA nanoparticles," Journal of Controlled Release, vol. 112, no. 1, pp. 88-95, 2006.

[20] L.-Y. Huang, T.-Y. Liu, A. Mevold et al., "Nanohybrid structure analysis and biomolecule release behavior of polysaccharideCDHA drug carriers," Nanoscale Research Letters, vol. 8, article 417, 2013.

[21] Q. Hu, B. Li, M. Wang, and J. Shen, "Preparation and characterization of biodegradable chitosan/hydroxyapatite nanocomposite rods via in situ hybridization: a potential material as internal fixation of bone fracture," Biomaterials, vol. 25, no. 5, pp. 779$785,2004$.

[22] S.-M. Kuo, G. C.-C. Niu, C.-W. Lan, M.-F. Cheng, M.-Y. Chiang, and S.-J. Chang, "Guided tissue regeneration with use of $\mathrm{CaSO}_{4}$-chitosan composite membrane," Journal of Medical and Biological Engineering, vol. 29, no. 6, pp. 304-310, 2009.

[23] H. Y. Wang, H. Qian, and W.-R. Yao, "Melanoidins produced by the maillard reaction: structure and biological activity," Food Chemistry, vol. 128, no. 3, pp. 573-584, 2011. 
[24] F.-H. Lin, C.-H. Yao, J.-S. Sun, H.-C. Liu, and C.-W. Huang, "Biological effects and cytotoxicity of the composite composed by tricalcium phosphate and glutaraldehyde cross-linked gelatin," Biomaterials, vol. 19, no. 10, pp. 905-917, 1998.

[25] A. M. C. Barradas, H. Yuan, C. A. van Blitterswijk, and P. Habibovic, "Osteoinductive biomaterials: current knowledge of properties, experimental models and biological mechanisms," European Cells \& Materials, vol. 21, pp. 407-429, 2011. 

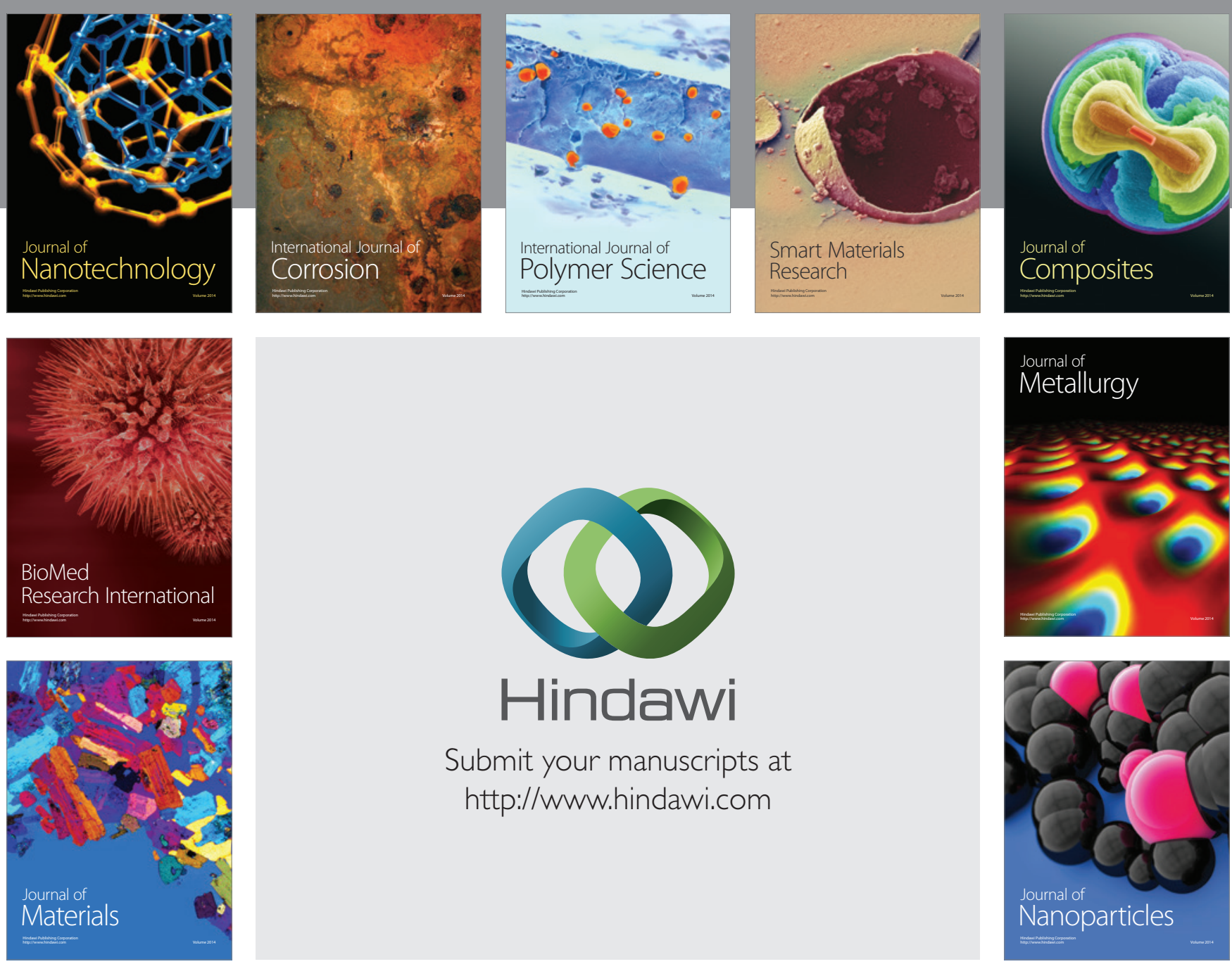

Submit your manuscripts at http://www.hindawi.com
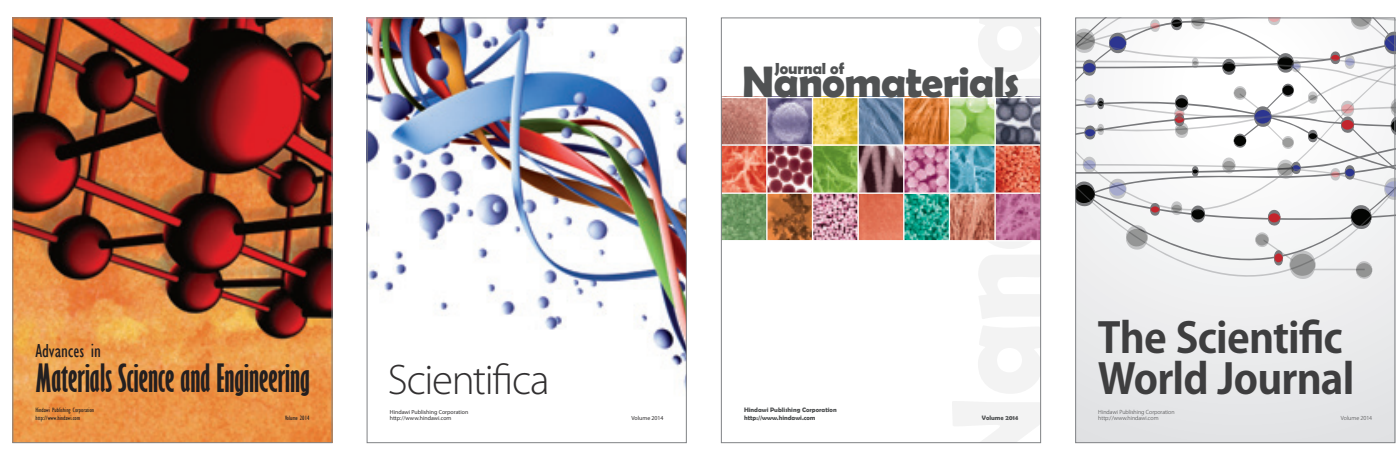

\section{The Scientific World Journal}
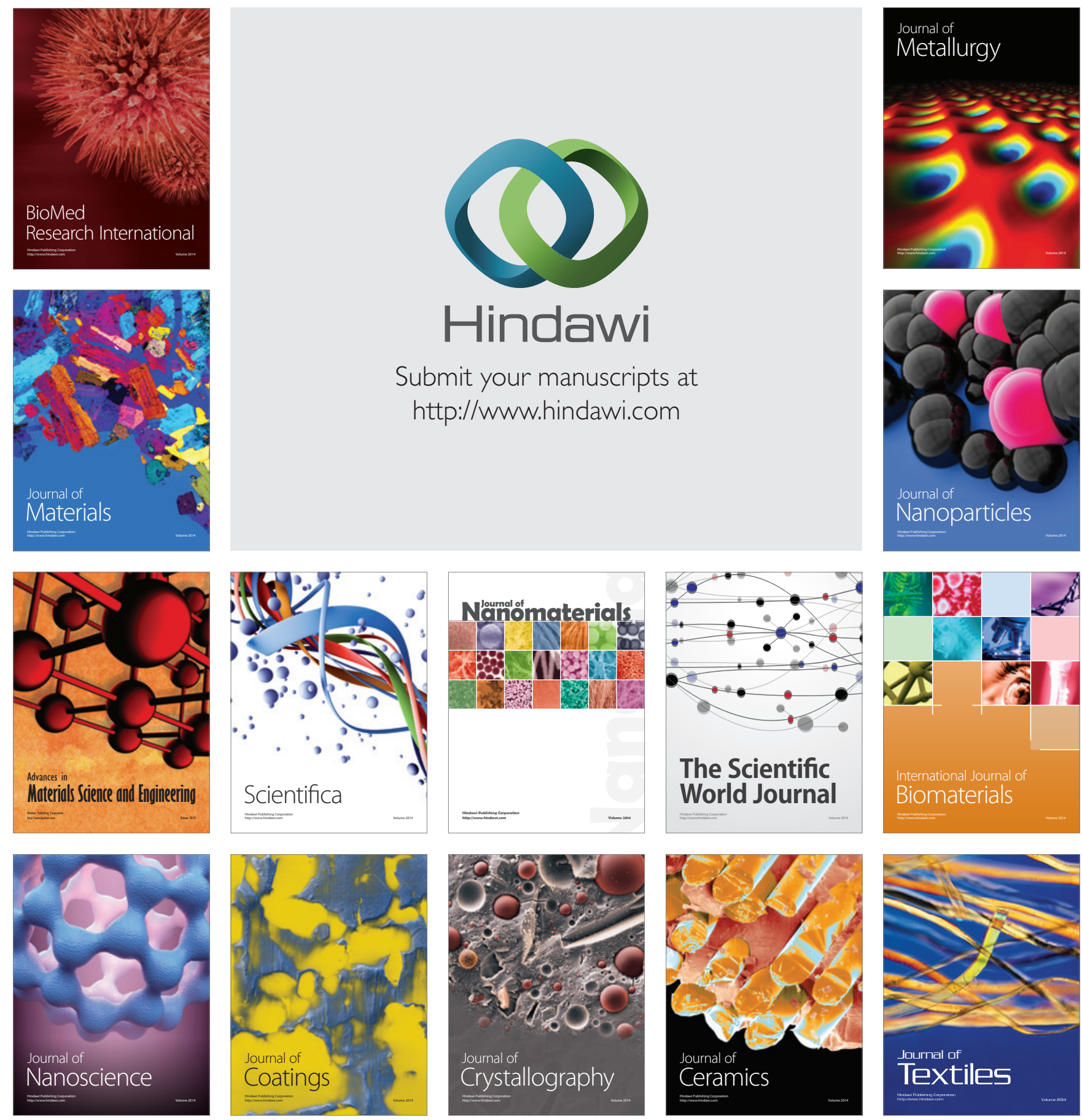